Joumal of the Mathematical Society of Japan Vol. 1, No. 3, Dec. 1949.

\title{
On the faithful representations of Lie groups
}

\section{Yozô Matushima}

(Received May 15, 1948)

It is well known that any Lie algebra may be represented faithfully by matrices. This result, which was first established by I. Ado, was by his powerful method proved by E. Cartan. ${ }^{2)} \mathrm{K}$. Iwasawa ${ }^{3)}$ has recently given purely algebraic proof; His result is most general in the sense that he proved the theorem for Lie algebras over an arbitrary field. But Cartan's analytical method of proof is a most direct one and has an advantage enabling us to discuss simultaneously the problem in the large. In the present paper we first attempt to simplify the Cartan's proof. Namely we construct a Lie algebra (which we shall call normal) containing the given algebra and having a more simple structure than the given one. Hence the problem is reduced to that of the faithful representations of normal Lie algebras and this may readily be reduced to nilpotent case by Cartan's method. Thus we may proceed without the rather complicated arguments in solvable and general cases. Further we prove some results on the faithful representations of Lie groups in the large. Some of them were already obtained by F. Cartan ${ }^{4}$ and A. Malcev. ${ }^{5}$ )

$\S$ 1. Let $L$ be a Lie algebra over a field P. A linear mapping $d$ on $L$ will be called a derivation if

$$
d[x, y]=[d x, y]+[x, d y]
$$

The mapping $d_{a}: x \rightarrow[\alpha, x]$ is, as we may readily verify, a derivation which we shall call inner derivation defined by an elemeut $a \in L$.

In the following we assume that $P$ is of characteristic 0 .

Lemma 1.6) Let $d$ be a derivation of $L$. Then we may represent $d$ uniquely in the form

$$
\text { (1) } \quad d=d^{o}+d^{s}, \quad d^{o} \quad d^{s}=d^{s} \quad d^{o} \text {, }
$$

1) Ado [I]

2) Cartan [3]

3) Iwasawa [1]

4) Cartan [6]

5) Malcev [3]. Malcev's papers [2] and [3] are not yet accessible to the writer. I knew his results on reading Mathematical Reveiews. Some were also obtained in M. Gotô [2] independently.

(6) Gantmacher [1] 
where $d^{o}$ and $d^{s}$ are derivations which are, as the linear mappings on $L$ nilpotent and semi-simple ${ }^{7}$ respectively. If $M$ is a linear subspace in $L$ such that $d M \subseteq M$, then $d^{o} M \subseteq M$. Moreover if $d^{\prime \prime}$ is a derivation such that $d d^{\prime}=d^{\prime} d$, then we have $d^{\prime} d^{o}=d^{o} d^{\prime}$ and $d^{\prime} d^{s}=d^{s} d^{\prime}$.

Proof. First let $P$ be algebraically closed. We decompose $L$ into the eigen-spaces of $d: L=L_{\alpha}+L_{\beta}+\ldots \ldots$, where $L_{\beta}$ mean the eigen-spaces corresponding to the eigen-values $\rho(\rho=\alpha, \beta, \ldots \ldots)$. Then $\left[L_{p} L_{\sigma}\right] \subseteq L_{p+\sigma}$, where $L_{\rho+\sigma}=0$ if $\rho+\sigma$ is not an eigen-value. Now we define the linear mapping $d^{s}$ by

$$
d^{s} x=\rho x, \text { for } x \in L_{p}(\rho=\alpha, \beta, \ldots \ldots) \text {. }
$$

We may easily see that $d^{s}$ is a semi-simple derivation such that $d d^{s}=d^{s} d$ and $d=d-d^{s}$ is nilpotent. Then $d=d^{s}+d^{s}, d^{o} d^{s}=d^{s} d^{\circ}$. The uniqueness and the other propositions may easily be seen from the above construction. Next let $P$ be arbitrary and $K$ be the finite Galois extension containing all the characteristic roots of $d$. Since $d$ is also a derivation of $L_{K}$, we may decompose $d$ as above into the form (1), where $d$ and $d^{s}$ are derivations of $L_{K}$. Let $e_{1}, \ldots \ldots, e_{r}$ be a basis of $L$ over $P$ and $a$ a derivation of $L_{K}$ defined by

$$
\alpha \cdot e_{i}=\sum_{k=1}^{r} e_{k} \alpha_{k i}, \alpha_{k i} \in K .
$$

Since the structural constants corresponding to the basis $e_{1}, \ldots, \ldots, e_{r}$ belong to $P$, the linear mapping $\theta a$ defined by

$$
\theta \quad a \cdot e_{i}=\sum_{k=1}^{r} e_{k}\left(\theta u_{k i}\right)
$$

is also a derivation, where $\theta$ denotes a substitution of the Galois group of $K / P$. Hence $\theta d=d=\theta d^{\circ}+\theta d^{s}$ and clearly $\theta d^{\circ} \cdot \theta d^{s}=\theta d^{s} \cdot \theta d$. Morcover, as we may easily see, $\theta d^{\circ}$ and $\theta d^{s}$ are nilpotent and semi-simple respectively. Hence we have $\theta d^{\prime \prime}=d^{\circ}$ and $\theta d^{s}=d^{s}$ for any $\theta$ by the uniqueness of the decomposition (1) and this shows that $d^{o}$ and $d^{s}$ are derivations of $L$.

Lemma 2. Let $R$ be a solvable ideal in $L$ and $N$ the maximal nilpotent ideal in $R$. Then $[L, R] \subseteq N$.

Proof. Let $R_{1}$ and $N_{1}$ be the radical and the maximal nilpotent ideal in $L$. Then $\left[L, R_{1}\right] \subseteq N_{1}{ }^{8}{ }^{8} \quad$ Hence $[L, R] \subseteq R \cap N_{1} \subseteq N$.

Definition. Let $L=S+R, S \cap R=0$ be a Levi decomposition of $L$, where $S$ and $R$ denote a semi-simple subalgebra and the radical of $L$ respectively. We call $L$ normal, if $R$ contains a nilpotent ideal $N$ in $L$ and

7) A linear transfomation is called semi-simple, if it has simple elementary divisors.

8) Cartan [1] P. 108 or Iwasawa [1], Matsushima [1] 
an abelian subalgebra $A$ such that $R=A+N, A \cap N=0,[S, A]=0 .^{\text {?) }}$

Theorem 1. We may construct a normal Lie algcbra $L_{1}$ containing the given Lie algebra $L$ such that every ideal in $L$ is also an ideal in $L_{1}$ and

$$
L_{1}=A+L, A \cap L=0 \text {, }
$$

where $A$ is an abelian subalgebra.

Proof. Let $S, R$ and $N$ be a maximal semi-simple subalgebra, the radical and the maximal nilpotent ideal in $L$ respectively. $R$ is a completety reducible S-module by the well known complete reducibility of representations of semi-simple Lie algebras. Hence there exists an S-module $M$ such that $R=M+N$. Since $[S, R] \subseteq N$ by Lemma 2 , we have $[S, M]=0$. Let $h$ be a regular element of $R$, i.e. an element which has as many different eigen-values as possible in the regular representation of $R$, and let $h=h_{1}$ $+h_{2}, h_{1} \in M, h_{2} \in N$. We may easily see that $h_{1}$ is also regular and $\left[h_{1}\right.$, $S]=0$. Let $\varphi(x)=x^{-l} \varphi_{1}(x)$ be the characteristic polynomial of the inner derivation $d=d_{h_{1}}$. where $\left(x, \varphi_{1}(x)\right)=1$. Let $R_{0}$ and $R_{1}$ be the linear subspaces in $R$ composed of all elements $x \in R$ such that $d^{l} x=0$ and $\varphi_{1}(d) x=$ 0 respectively. Then $R=R_{0}+R_{1}$ and since $d$ induces in $R$ a non-singular linear mapping, $d R_{1}=\left[h_{1}, R_{1}\right]=R_{1}$, whence $R_{1}$ is contained in $N$ by Lemma 2. As is well known, $R_{0}$ is a nilpotent subalgebra. Furthermore since $d S$ $=0$, we have $\left[S, R_{0}\right] \subseteq R_{0}$ and $\left[S, R_{1}\right] \subseteq R_{1}$. Let $N_{0}=R_{0} \cap N$. Then $N_{o}$ is an S-module and there exists an S-module $M_{0}$ such that $R_{0}=M_{0}+N_{0}$. We have as above $\left[S, M_{0}\right]=0$. As we may easily see, $N=N_{0}+R_{1}$. Let $a_{1}, \ldots$ $\ldots, a_{m}$ be a basis of $M_{0}$ over $P$. We decompose the inner derivations $d_{a_{i}}$ into the form (1) and put $d_{i}=d_{a_{i}}$. Then we have $d_{i} x=0$, for $x \in S$ or $x$ $\epsilon R_{0}$, because $d_{a_{i}}$ induce in $S+R_{0}$ nilpotent linear transformations. Then $d_{i} d_{a_{j}} x=d_{i}\left[a_{j} x\right]=\left[a_{j}, d_{i} x\right]=d_{a_{j}} d_{i} x$ for all $x \in L$, whence $d_{i} d_{a_{j}}=d_{a_{j}} \cdot d_{i}$. Therefore $d_{i} d_{j}=d_{j} d_{i}(i, j=1, \ldots \ldots, m)$ by Lemma 1 . Let now $A$ be the abelian Lie algebra spanned by the derivations $d_{1}, \ldots \ldots, d_{m}$ and let $L_{1}=A+$ $L$ be the direct sum of two vector spaces $A$ and $L$. We define the multiplication between elements of $L_{1}$ as follows :

$$
\left[u_{1}+x_{1}, u_{2}+x_{2}\right]=u_{1} x_{2}-u_{2} x_{1}+\left[x_{1} x_{2}\right]
$$

where $u_{i} \in A$ and $x_{i} \in L(\mathrm{i}=1,2)$ and $u_{i} x_{j}$ mean the element of $L$ which are the images of $x_{j}$ under the derivations $u_{i}$. We may easily verify that $L_{1}$ becomes a Lic algebra. Furthermore $A$ is an abelian subalgebra and

9) Normal Lie algebras have the similar structure to algebraic Lie algebras. For algebraic Lie algebras, see Chevalley and Tuan [1], Gotô [1], Matsushima [1] 
every ideal in $L$ is also an ideal in $L$ by Lemma 1. Clearly $A+R$ is the radical of $L_{1}$ and $A+R=A+M_{0}+N$. Let $N_{1}=P\left(a_{1}-d_{1}\right)+\cdots \cdots+P\left(a_{m}-\right.$ $\left.d_{m}\right)+N$. then $A+R=A+N_{1}$. We easily verify that $N_{1}$ is an ideal in $L_{1}$. Moréover, since $N_{1}$ is solvable and $N$ is nilpotent and every eigenvalue of $a_{i}-d_{i}$ in the adjoint representation is $0, N_{1}$ is nilpotent. Further we have $[S, A]=0$. Thus $L_{1}$ is normal.

Remark. In the above proof, we used the semi-simplicity of $S$ only in the point that $R$ is a completely reducible S-module. Further we may prove that $N_{1}$ is the maximal nilpotent ideal and the derived algebra of $L_{1}$ coincides with that of $L$.

$\S 2$. In this section we apply Theorem 1 on the proof of faithful representability of Lie algebras. It is suffcient by Theorem 1 to prove the problem for normal Lie algebras. Let $L$ be a normal Lie algebra over the field $P$ of all real or complex numbers and let

$$
L=S+A+N, \quad A+N=R, \quad[S, A]=0,
$$

as in $\S 1$. Then $T=S+A$ is a subalgebra in $L$, whose center equals $A$. Let $\mathfrak{N}$ and $\mathfrak{I}$ be local subgroups in $\mathfrak{R}$, which correspond to the subalgebras $N$ and $T$ respectively, where $\mathfrak{Q}$ denotes the Lie group whose Lie algebra is $L$. Then $\mathfrak{R}$ is a nilpotent invariant subgroup and we may introduce canonical coordinates $(x)$ in $\mathfrak{R}$ such that, if $x^{\prime}=a x$, where $a$ and $x$ denote the elements of $\mathfrak{R}$ whose coordinates are $(a)$ and $(x)$ respectively, then $x^{\prime}{ }_{i}=g_{i}(a, x)$ are polynomials of $(a)$ and $(x)$. Moreover

$$
\mathfrak{L}=\mathfrak{N T}, \quad \mathfrak{R} \cap \mathfrak{I}=1 .
$$

Hence every element of $\mathfrak{Q}$, which is sufficiently near the unit element, may be uniquely represented in the form $a b, a \in \mathfrak{N}, b \in \mathfrak{I}$. Then $(a b)(x y)=$ $x^{\prime} y^{\prime}$, where

$$
x^{\prime}=a\left(b \times b^{-1}\right), y^{\prime}=b y, x, a \in \mathfrak{R} ; \quad b, y \in \mathfrak{T} .
$$

As we may readily verify, the transformations $(x) \rightarrow\left(x^{\prime}\right)$ of the coordinates in $\mathfrak{R}$ defined by (2) form a transformation group $\mathbb{S}$ homomorphic to $\mathfrak{B}$. Moreover the elements of $\mathfrak{R}$, which correspond to the unit transformation of $\mathbb{B}$, are contained in $\mathfrak{T}$. Hence

$$
\text { (S) } \equiv \mathfrak{R} / \mathfrak{D}
$$

where $\mathfrak{D}$ is an invariant subgroup contained in $\mathfrak{T}$. We represent the transformation in (S) defined by (2) by the equations

$$
x^{\prime}{ }_{i}=F^{i}(a b ; x) \text {. }
$$

10) Cartan [3] 
Let $f(x)=b x b^{-1}$. Then, since the coordinates are canonical,

$$
f_{i}(x)=\Sigma_{k} \alpha_{i k}(b) x_{k} \text {. }
$$

and we have $F_{z}(a b ; x)=g_{i}(a ; f(x))$. As $g_{i}(a ; f(x))$ are polynomials of $(x)$. Hence we may faithfu!ly represent $\left(\&\right.$ by matrices by Cartan's lemma. ${ }^{10}$ Hence there exists a representation $R_{1}$ of $L$ such that $R_{1} \cong L / D$, where $D$ denotes the ideal in $L$ which corresponds to the invariant subgroup $D$ in $\mathfrak{L}$. That is clearly a faithful representation $R_{2}$, because it is the direct sum of semi-simple ideal and its centre. Since $T^{\prime}=L / N, R_{2}$ is also a representation of $D$. Then, as $D$ is contained in $T$, the representation $R$ of $L$ defined by

is faithful. Thus we proved the

$$
R=\left[\begin{array}{ll}
R_{1} & \\
& R_{2}
\end{array}\right]
$$

Theorem 2. Any Lie algebra $L$ over the ficld $P$ of real or complex numbers may be represinted faithfully by matrices.

$\S 3$. Next we investigate the problem of faithful representability of Lie groups in the large. Let $\mathfrak{N}$ be a simply connected nilpotent Lie group. The parameters of $\mathfrak{N}$ may be real or complex. $\mathfrak{N}$ is homeomorphic with an euclidean space and we may introduce coordinates in the large in $\mathfrak{N}$ such that they are canonical in the neighbourhood of the unit element and if $x^{\prime}=a x$, then the coordinates of $x^{\prime}$ are polynomials of $a$ and $x^{10)}$. Let $\theta$ be an antomorphism of $\mathfrak{N}$ and let $x_{i}^{\prime}=\theta_{i}(x)$, where $\theta_{i}(x)$ are coordinates of $\theta(x)$. If $(x)$ is sufficiently small, then, since the coordinates are canonical, $x_{i}^{\prime}=\sum_{j} \bar{\theta}_{i j} x_{j}$. Now we define the mapping $\bar{\theta}$ of $\mathfrak{N}$ onto itself by the equations

$$
x_{i}^{\prime}=\sum_{j} \theta_{i j} x_{j}
$$

and show that $\theta=\theta$. For, since $\theta=\bar{\theta}$ in the neighbourhood of $(0) . \theta(a b)$ $=\ddot{\theta}(a) \dddot{\theta}(b)$ for sufficiently small $(a),(b)$. But as the coordinates of $\theta$ $(a \dot{b})$ and $\bar{\theta}(a) \cdot \ddot{\theta}(b)$ are polynomials of $(a)$ and $(b)$ respectively, $\bar{\theta}(a b)$ $=\theta(a) \cdot \theta(b)$ holds for any $a$ and $b$. Thus $\theta$ is an automorphism of the connected group $\mathfrak{N}$ and we have $\theta=\bar{\theta}$, since they coincide in the neighbourhood of the unit element. Thus every automorphism of $\mathfrak{N}$ is represented (in our coordinate) by linear transformation. Then we may prove the following Lemma by the similar argument as in the proof of Theorem 2.

Lcmma 3. Let $\mathfrak{L}$ be a lie group and let $\mathfrak{I}$ and $\mathfrak{N}$ be a closed subgroup and a closed simply connected nilpotent invariant subgroup respe-

10) Cartan [3]. 
ctively such that

$$
\mathfrak{Z}=\mathfrak{I N} \quad \mathfrak{I} \cap \mathfrak{R}=\mathbf{1}
$$

If $\mathfrak{I}$ is faithfully representable ( $f . r$.$) , then \mathbb{B}$ is also f.r.

Now we may prove the following

Theorem 3. Any simply connected complex Lie group $\mathfrak{L}$ is f.r.

Proof. Let $L$ be the Lie algebra of $\mathfrak{Q}$ and $L_{1}$ the normal Lie algebra containing $L$ constructed in $\S \mathbf{1}$. Then $L_{x}=A+L$ and the simply connected Lie group $\mathfrak{R}_{1}$ corresponding to $L_{1}$ contains $\mathfrak{L}$ as a closed subgroup. ${ }^{11)}$ Hence we may assume that $L$ itself is normal. Then $L=S+A+N$ as in $\S 1$ and $L$ contains simply connected closed subgroups $\mathfrak{I}$ and $\mathfrak{R}$ whose Lie algebras are $S+A$ and $N$ respectively. ${ }^{12} \quad$ Moreover $\mathfrak{L}=\mathfrak{T} \mathfrak{N}, T \cap \mathfrak{N}=1$ and $\mathfrak{R}$ is nilpotent and invariant and $\mathfrak{I}$ is the direct product of a simply connected complex semi-simple group and a vector group. Since connected complex semi-simple groups are f.r. by a theorem of Cartan ${ }^{12)}$ and vector groups are also f.r., the same holds for $\mathfrak{T}$. Then $\mathfrak{L}$ is f.r. by Lemma 3 .

Remark. If $\mathbb{R}$ is solvable, as we may see from the above proof, the condition that $\mathfrak{R}$ is of complex parameters is unnecessary.

Theorem 4 . Let $\&$ be a connected Lie group and $L$ its Lie algebra. Suppose that $\mathfrak{Q}$ contans connected closed subgroups $\mathfrak{I}$ and $\mathfrak{R}$ which satisfy the following conditions:

1) $\mathfrak{R}$ is a simply connected solvable invariant subgroup and $\mathfrak{I}$ is f.r.

2) $\mathfrak{L}=\mathfrak{T} \mathfrak{R}, \quad \mathfrak{I} \cap \mathfrak{R}=\mathbf{1}$.

3) Denote bv $T$ and $R$ the subalgebras of $L$ corresponding to $\mathfrak{I}$ and $\mathfrak{R}$ respectively. Then $R$ is a completely reducible $T$-module. ${ }^{13)}$ Then $\mathfrak{R}$ is f.r.

Proof. By the remark in $\S 1$. we may construct a Lie algebra $L_{1}$ containing $L$ as an ideal such that

$$
L_{1}=A+L=T+R+A . \quad A \cap L=0, \quad[A T]=0 .
$$

where $A$ is an abelian subalgebra. Moreover $A+R$ is a solvable ideal in $L$ and $A+R=A+N$, where $N$ is a nilpotent ideal in $L$. We construct a Lie group $\mathfrak{R}_{1}$ whose Lie algebra is $L_{1}$ such that it contains $\mathfrak{L}$ as a closed subgroup and satisfies the conditions of Lemma 3 . Denote by $\mathfrak{A}$ the vector group whose Lie algebra is $A$. Let $\mathfrak{Q}_{1}$ be the product space of $\mathfrak{T} \times \mathfrak{A}$ and $\mathfrak{R}$, where $\mathfrak{I} \times \mathfrak{A}$ denotes the direct product of the groups $\mathfrak{T}$ and $\mathfrak{A}$. We

11) Pontrjagin [1] Chap. IX $\S 54$

12) Cartan [2]

13) The condition 3) is satisfied, if $\mathfrak{I}$ is compact or semi-simple. 
will define a continuous automorphism $\varphi_{x}$ of $\mathfrak{R}$ for each $x \in \mathfrak{I} \times \mathfrak{X}$ in such a way that $\varphi_{x y}=\varphi_{x} \varphi_{y}$ holds. Put $\varphi_{t}(r)=\operatorname{tr} t^{-1}$ for $t \in \mathfrak{I}, r \in \mathfrak{R}$. Let next $\mathfrak{R}_{1}^{\prime}$ be the local Lie group corresponding to $L_{1}$. Then $\mathfrak{R}_{1}^{\prime}=$ $\left(\mathfrak{I}^{\prime} \times \mathfrak{A}^{\prime}\right) \mathfrak{R}^{\prime} . \quad\left(\mathfrak{I}^{\prime} \times \mathfrak{U}^{\prime}\right) \cap \mathfrak{R}^{\prime}=1$, where $\mathfrak{I}^{\prime}$ etc. may be considered as the neighbourhoods of the unit element of $\mathfrak{I}$ etc. Let $\varphi_{a^{\prime}}\left(r^{\prime}\right)=a^{\prime} r^{\prime} a^{\prime-1}$, where $a^{\prime} \in \mathfrak{H}^{\prime}, \quad r^{\prime} \in \mathfrak{R}^{\prime}$. Since $\mathfrak{R}$ is simply connected, we may extend $\varphi_{a^{\prime}}$ to a continuous automorphism of $\mathfrak{R}$. More over we may define a continuous automorphism $\varphi_{a}$ for each $a \in \mathfrak{A}$ by the argument given in Pontrjagin [1] p. 275-276. Clearly $\varphi_{x y}=\varphi_{x} \varphi_{y}$ for $x, y \in \mathfrak{I}$ or $x, y \in \mathfrak{A}$. We show that

$$
\varphi_{t}\left(\varphi_{a}(r)\right)=\varphi_{a}\left(\varphi_{t}(r)\right), t \in \mathfrak{T}, \quad a \in \mathfrak{A}, \quad r \in \mathfrak{R} .
$$

Since each group is connected, it is sufficient to prove the above relation for $t \in \mathfrak{T}^{\prime}, a \in \mathfrak{A}^{\prime}, r \in \mathfrak{R}^{\prime}$. Then

$$
\varphi_{t}\left(\varphi_{a}(r)\right)=t\left(a r a^{-1}\right) t^{-1}=(t a) r(t a)^{-1}=(a t) r(a t)^{-1}=\varphi_{a}\left(\varphi_{t}(r)\right)
$$

Put $\varphi_{r}(r)=\varphi_{t}\left(\varphi_{a}(r)\right)$ for $x=t a \in \mathfrak{I} \times \mathfrak{A}$, where $t \in \mathfrak{I}, a \in \mathfrak{A}$. Then clearly $\varphi_{x y}=\varphi_{x} \varphi_{y}$. We now define the product of two pairs $\left(r_{1}, x_{1}\right),\left(r_{2}, x_{2}\right)\left(r_{i} \epsilon\right.$ $\left.\in \mathfrak{R}, x_{i} \in \mathfrak{I} \times \mathfrak{A}\right)$ of the set $\mathfrak{L}_{1}$ as follows :

$$
\left(r_{1}, x_{1}\right) \quad\left(r_{2}, x_{2}\right)=\left(r_{1} \varphi_{x_{1}}\left(r_{2}\right), x_{1} x_{2}\right) .
$$

Then $\mathfrak{Q}_{1}$ becomes a Lie group whose Lie algebra is $L_{1}{ }^{11)}$. Clearly $\mathfrak{Q}$ is a closed invariant subgroup in $\mathfrak{l}_{1}$. Furthermore $\mathfrak{R}_{1}=(\mathfrak{I} \times \mathfrak{A}) \mathfrak{R},(\mathfrak{I} \times \mathfrak{A}) \cap \mathfrak{R}$ $=1$ and, as we may see from the above construction $\mathfrak{A} \mathfrak{R}$ is a simply connected subgroup whose Lie algebra is $A+R$. Since $A+R=A+N$. $\mathfrak{A} \mathfrak{R}$ contains simply connected nilpotent subgroup $\mathfrak{N}$ such that $\mathfrak{X} \mathfrak{R}=\mathfrak{U} \mathfrak{N}$, Then $\mathfrak{l}_{1}=(\mathfrak{I} \times \mathfrak{P}) \mathfrak{N}$ and since $(\mathfrak{I} \times \mathfrak{U}) \cap \mathfrak{U} \mathfrak{R}=\mathfrak{U}, \quad(\mathfrak{I} \times \mathfrak{U}) \cap \mathfrak{R}=(\mathfrak{I} \times \mathfrak{A}) \cap \mathfrak{U} \mathfrak{R} \cap$ $\mathfrak{N}=\mathfrak{A} \cap \mathfrak{N}=1$. Further $\mathfrak{I} \times \mathfrak{U}$ is f.r. Thus $\mathfrak{L}_{1}$ satisfies the conditions in Lemma 3 and is therefore f.r. Since $\mathfrak{R}_{1} \supset \mathfrak{L}$, the same is true for $\mathfrak{L}$.

Remark. Theorem 3 follows also from this theorem.

From Theorem 4 and a theorem of A. Malcev ${ }^{14)}$ on the structure of linear solvable Lie groups we obtain immediately the following

Theorem 5..$^{15)}$ A connectid solvable Lie group $\mathfrak{S}$ is f.r. if and only if it contains a connected compact abelian group $\mathfrak{A}$ and a closed simply connected invariant subgroup $\mathfrak{R}$ such that

$$
\mathfrak{L}=\mathfrak{A} \mathfrak{R}, \quad \mathfrak{A} \cap \mathfrak{R}=1 .
$$

\section{Nagoya University}

14) Maldev [1], [2] Gotô [2।

15) Malcev [3], Gotô [2] 


\section{References}

\section{Ado}

[1] Über die Darstellnng der endlichen kontinuierlichen Gruppen durch lineare Substitution Bull Soc. Physico Math. de Kazan, 7 (1935)

E. Cartan

[1] Thèse, Paris (1894)

[2] La topologie des groupes de Lie, Exposés de Geometrie VIII. (1936)

[3] Les représentations linéaires des groupes de Lie, Jour. de Math. pures et appliquées, t. 17 (1938)

C. Chevalley and H. F. Tuan

[1] On algebraic Lie algebras, Proc. Nat Acad. Sci. U.S.A 31 (1945)

F. Gantmacher

[1] Canonical representation of automorphisms of a complex semi-simple Lie group, Rec. Math. 5 (1939)

M. Gotô

[1] On algebraic Lie algebras, Jour. Math. Soc. Japan, 1 (1948)

[2] Faithful representations of Iie groups, Matematica Japonicae, (1949) K. Iwasawa

[1] On the representation of Lie algebras, Jour. Math., 19 (1948)

A. Malcev

[1] Subgroups of Lie groups in the large, C. R. d'Acad. URSS (1942)

[2] On the theory of Lie groups in the large, Rec. Math. 16 (1945)

[3] On linear Iile groups, C. R. d Acad. URSS 40 (1943)

Y. Matsushima

[1] On algebraic Lie groups and algebras, Jour. Math. Soc. Japan, 1 (1948)

L. Pontrjagin

[1] Topological groups, Princeton (1939) 